\title{
Le caractère éthique du jugement médical
}

\author{
L. AYACHE \\ Villa du palmier, 203, rue Marcel Vandelli, 83200 Le Pradet
}

Les progrès récents et les développements prévisibles des techniques médicales d'assistance à la sexualité, touchant notamment la fonction érectile et la maîtrise de l'éjaculation, conduisent certains spécialistes à s'interroger sur la composante éthique de la définition des indications de ces traitements. La diffusion sans discernement de procédés assurant la performance sexuelle en toute circonstance ne risque-t-elle pas de modifier profondément le rapport de la sexualité au désir et au sentiment à tel point que l'avantage apparent de ces techniques menacerait une dimension de notre humanité? Platon, dans un passage bien connu du Banquet, dressait déjà par la bouche d'Aristophane le tableau d'une humanité mythique effrayante dans laquelle chaque individu, ayant retrouvé sa moitié, s'y accolerait en une copulation perpétuelle, chaque couple fusionnant en une boule indissociable [1]. Le manque, le désir, la quête et l'insatisfaction sont des aspects essentiels de notre humanité. La plénitude est inhumaine.

Il est aujourd'hui devenu banal de mettre en exergue les risques qu'engendrent indirectement les progrès techniques, quels que soient les bénéfices qu'on en espère dans l'immédiat. On renvoie souvent, pour rendre compte de cette ambivalence des moyens techniques que les hommes se donnent, à une distinction entre, d'une part, la science et la technique qui offrent des possibilités en elles-mêmes innocentes et, d'autre part, l'usage que certains hommes en font, lequel seul serait moralement louable ou condamnable. Une illustration récurrente de cette distinction oppose les usages médicaux et les usages militaires des rayonnements ionisants. Pour concilier le progrès technique et les exigences morales, il faudrait, selon cette problématique qui tend à devenir désuète, imposer en aval des découvertes scientifiques et techniques une régulation morale de leur utilisation. Deux axes de réflexion s'imposent alors : selon quels principes interdire ou permettre les usages d'une technique nouvelle? comment faire respecter ces mesures? La première question se subdivise elle-même en deux problèmes, l'un proprement moral, celui des principes, l'autre, concernant l'application de ces principes, relevant de la casuistique. La seconde question est une question politique.

La régulation morale des inventions techniques selon cette manière d'envisager les choses se heurte à des obstacles difficiles à surmonter dans les trois registres que j'ai mentionnés. D’abord, il n'y a pas de consensus sur les principes moraux. Ensuite, le jugement qui applique un principe moral sur un cas particulier est toujours discutable. Enfin, la mondialisation du savoir et des techniques rend difficile le respect des interdictions édictées le plus souvent à l'échelle des États. Cependant, il faut reconnaître que, malgré ces difficultés, la seconde partie du $\mathrm{XX}^{\mathrm{e}}$ siècle a progressé dans chacun de ces domaines : depuis la seconde guerre mondiale se dessine un certain " consensus par recoupement "[2] relatif aux droits de l'homme; la discussion des bénéfices et des risques à attendre de la mise en œuvre 
des nouvelles techniques, notamment médicales, se généralise et, dans les pays développés, s'institutionnalise (comités d'éthique); enfin, la mondialisation de l'information suscite l'émergence d'une opinion publique transnationale qui conduit à une forme du droit d'ingérence s'opposant à l'apparition de paradis (ou d'enfers!) techniques (comparables aux paradis fiscaux) dans lesquels tout ce que les pays développés s'interdisent serait toléré.

Même si les difficultés dans les registres des principes, de la casuistique et de la politique peuvent être, sinon surmontées, du moins affrontées par la recherche de consensus par recoupement, la discussion au sein de comités d'éthique et le recours à des institutions internationales pour l'application de résolutions politiques, cette manière de régler l'application technique des découvertes scientifiques demeure à mon sens fondamentalement défectueuse parce qu'elle repose sur une position incorrecte du problème. En effet, la recherche scientifique et technique ne peut être disjointe de la question de son usage et, de ce fait, on ne peut simplement distinguer des applications légitimes et des utilisations condamnables des nouveaux possibles engendrés par la science. Le recul dont nous disposons à présent relativement aux conséquences des innovations techniques montre que le danger réside moins dans un usage délibérément nuisible d'une découverte que dans l'absence de contrôle de l'enchaînement immense des conséquences d'une action technique quelle que soit l'intention qui en est à l'origine. Cet enchaînement causal se révèle souvent pour une part circulaire, au point que les conséquences lointaines d'une action peuvent annuler son effet immédiat voire aggraver la situation initiale. L'induction de gènes résistants par l'usage massif d'antibiotiques est un exemple bien connu de cette rétroaction inverse d'une pratique technique. L'évaluation d'un procédé ne peut donc plus se réduire à la balance entre l'avantage résultant d'un usage profitable et les risques d'un dévoiement de son utilisation : elle doit tenter de pondérer les avantages immédiats et les effets lointains et incertains que son intervention peut engendrer dans l'équilibre dynamique et instable des écosystèmes, des ensembles sociaux, et même de la totalité psychosomatique que constitue chaque personne. Ainsi, une technique aussi profitable aux hommes que la vaccination dont l'efficacité est incontestable, du fait même de sa réussite (je pense notamment à l'éradication de la variole), peut entraîner, par modification de la "pathocénose ", un déséquilibre de la concurrence entre germes pathogènes, une modification de la virulence de certains d'entre eux et une répartition nouvelle de lincidence des diverses maladies infectieuses, laquelle peut se révéler dommageable, en définitive, pour l'espèce humaine [3]. De même, le mécanisme naturel du rapport entre une espèce hôte et une espèce parasite conduit spontanément à la sélection des hôtes les plus résistants et des parasites les moins virulents ; or, ce mécanisme est entravé par les soins prodigués aux malades sans les guérir rapidement : en prolongeant la vie des patients, ces soins prolongent aussi celle des germes dont ils sont infectés et favorisent leur diffusion dans la population. On pourrait multiplier de tels exemples. Ils ne doivent pas conduire à une position malthusienne qui privilégierait l'intérêt de l'espèce au détriment de celui de l'individu. Il arrive en effet qu'à l'inverse, une pratique bénéfique au niveau d'une population se révèle nocive pour certains individus (on pourrait ici encore évoquer la vaccination). Il arrive en outre qu'une intervention réussie sur une partie du corps engendre des effets indésirables sur l'organisme considéré dans sa totalité ou sur la personnalité psychique du patient. Ces exemples montrent que l'évaluation d'une innovation technique exige la considération de divers niveaux de totalités qui s'englobent ou se recoupent partiellement et entretiennent des relations d'interaction complexes, parfois de rétroaction inverse. Ils doivent nous conduire à nous interroger sur la nature du jugement susceptible de conjuguer un tel ensemble immense de paramètres en tenant compte des degrés d'incertitude qui affectent diversement les facteurs à pondérer.

Or, un tel jugement ne relève ni de la technique, ni de la morale. La technique moderne est fondée, depuis le XVII ${ }^{\mathrm{e}}$ siècle, sur l'abstraction. C'est parce qu'elle ne considère pas la réalité concrète d'un événement singulier dans 
la totalité de son contexte, mais qu'elle construit un phénomène abstrait et reproductible en ne retenant qu'un nombre limité de paramètres quantifiables, que la science dégage des lois physico-mathématiques qui permettent une certaine maîtrise technique du réel. Même les branches les plus souples des mathématiques comme la théorie des probabilités sont impuissantes à considérer un ensemble indéfini de facteurs qui ne sont pas indépendants les uns des autres. La question des effets à long terme de la culture de plantes transgéniques, par exemple, n'est pas formulable dans le cadre de la discipline technique qui met au point de telles plantes, parce qu'il n'est pas possible de modéliser abstraitement l'interaction entre un gène et l'ensemble de l'écosystème qu'il affecte non seulement par une éventuelle contamination d'espèces parentes ou lors de son insertion dans la chaine alimentaire, mais aussi par la modification de la concurrence entre les espèces, qu'elles soient végétales ou animales, que produit la simple présence d'une nouvelle variété. Lorsque les effets d'une technique concernent de surcroît la réalité humaine dans ses aspects psychologique et sociologique, comme c'est le cas pour les techniques d'assistance à la sexualité, le jugement technique est encore plus largement débordé.

Le jugement moral n'est pas, lui non plus, pertinent pour formuler le problème des effets globaux d'une innovation technique. En effet, le jugement moral est par essence intransigeant. Il s'appuie sur une valeur considérée comme absolue et exige la conformité d'un acte avec cette valeur. Par exemple, il interdira toute atteinte à la dignité de la personne humaine. Or, même en supposant un accord universel sur certaines valeurs reconnues comme absolues, et même en supposant une procédure permettant de résoudre les questions de casuistique, ce problème demeurerait dans la plupart des cas inentamé par une investigation morale car il concerne le plus souvent un registre indifférent de ce point de vue. La morale peut ainsi servir de garde-fou pour éviter un usage délibérément nuisible de certaines techniques; elle ne permet pas de trancher la question de savoir si une action technique bien intentionnée sera à terme profitable ou non dans chaque circonstance.
Les questions soulevées par les nouvelles techniques d'assistance sexuelle montrent bien les limites d'une approche morale du problème. La morale peut aisément dissuader un médecin d'assister une personne dont les pratiques sexuelles seraient susceptibles d'être criminelles (en supposant que de tels comportements soient plus ou moins prévisibles et que l'amélioration de la fonction sexuelle aggrave ces pratiques, ce qui ne va pas de soi), mais elle ne peut décider de l'opportunité d'un traitement dans une situation moralement neutre dont je suppose qu'elle constitue le contexte habituel des consultations. Comment confronter l'exigence morale de dignité de la personne avec les questions de la sexualité chez l'adultère, l'adolescent, la personne âgée, l'homosexuel, etc.[4]? La morale est trop radicale pour régler des choix singuliers entre des options dont aucune ne met en cause ses exigences fondamentales ; lorsqu'elle s'aventure à prescrire jusqu'aux détails de la vie notamment sexuelle, elle devient dogmatique et plurielle. Or, rien ne serait plus immoral qu'une diversification des pratiques médicales selon l'obédience de chaque médecin. La régulation morale de l'activité médicale ne peut reposer sur d'autres principes que ceux qui sont susceptibles d'un « consensus par recoupement " garantissant une grande homogénéité des pratiques indépendamment des convictions de tel ou tel praticien. De tels principes d'une moralité commune sont suffisants pour exclure des pratiques extrêmes mais ils ne sauraient guider les choix quotidiens du médecin.

En dégageant ainsi un espace de jugement qui ne relève ni de la technique, ni de la morale, nous mettons le doigt sur le point douloureux de la modernité. En effet, un trait caractéristique de la modernité au moins jusqu'au milieu du XXe siècle réside dans l'illusion selon laquelle technique et morale couvriraient la totalité du champ des jugements prescriptifs. Le technicien prescrirait les moyens, le moraliste limiterait leur usage selon la valeur des fins en vue desquels ils sont mis en œuvre. Depuis Cl. Bernard, la médecine a ainsi coutume de s'adresser aux moralistes et aux hommes politiques pour qu'ils lui assignent les conditions d'application des procédés techniques, supposés en eux-mêmes innocents, 
qu'elle maîtrise au fur et à mesure des progrès scientifiques [5]. Le relatif échec de la régulation morale des techniques contribue à une inflation de l'exigence morale qui conduit de nos jours à une constante indignation. Jamais l'opinion publique ne s'est tant émue des injustices et des crimes. Notre monde n'est donc pas sans repère moral : au contraire, les récentes protestations contre les crimes pédophiles montrent que des pratiques naguère plus ou moins cachées et supportées deviennent aujourd'hui intolérables, non seulement parce qu'elles seraient plus courantes ou plus violentes que naguère, mais surtout parce qu'elles deviennent plus odieuses à l'opinion. Exacerbation de l'exigence morale, développement prodigieux des capacités techniques, relative inefficacité de la morale pour régler les techniques et les mœurs sont les trois ingrédients d'un monde moderne qui comporte, on l'admettra, quelque chose de tragique.

Si la modernité pose ainsi en des termes moraux et techniques des questions qui ne relèvent ni de la morale, ni de la technique, la question de la relative inefficacité des régulateurs éthiques des pratiques est susceptible de progresser en trouvant son registre propre. La réflexion éthique des dernières décennies va dans ce sens. Quel est donc le registre des questions évoquées plus haut : celles de l'impact à long terme des traitements par antibiotiques, d'une campagne de vaccination, de la culture de plantes transgéniques ou de l'assistance médicale dans le domaine de la sexualité?

Ce registre est celui de la prudence. La prudence ne qualifie pas seulement l'attitude de précaution qui consiste à minimiser les risques d'échec plutôt qu'à maximiser les chances de succès (ce qui suppose, comme on le sait, quelque audace). Elle désigne d'abord, dans un sens plus ancien et plus fort, la gestion parfaite des moyens d'une vie accomplie [6]. Dans une analyse célèbre de la pratique, E. Kant [7] montre que les prescriptions en vue du bonheur ne relèvent ni de la technique, ni de la morale. Il ne saurait être question d'une technique du bonheur, parce que le bonheur n'est pas un concept qu'on pourrait concevoir logiquement et dont on pourrait calculer les moyens à coup sûr, mais un idéal imaginaire mêlant des éléments contradictoires de façon différente pour chacun. Il ne saurait être question de morale lorsqu'il s'agit des moyens du bonheur, non parce que la morale n'aurait pas affaire au bonheur, mais parce qu'elle régit seulement la détermination de la volonté et n'est pas concernée par la recherche des moyens les plus efficaces pour la réalisation effective d'effets bénéfiques (c'est pourquoi chacun peut satisfaire à l'exigence morale selon Kant, quels que soient ses talents et son pouvoir de réussir ses entreprises, pourvu que sa volonté soit raisonnable). La prudence occupe précisément cet espace entre technique et morale : tournée vers mon profit exclusif, elle peut contrarier la morale, mise en ouvre pour un bonheur partagé, elle en est un moyen indispensable ; incapable, en raison de la diversité des idéaux de chacun et de la singularité des circonstances, de prendre la forme de règles techniques, elle formule des conseils qu'elle appuie, avec une certaine probabilité, sur l'expérience passée individuelle ou spécifique. Elle se nourrit des voyages et des discussions, tisse des analogies et des comparaisons entre des situations qu'elle envisage toujours globalement parce qu'elles ne se résument pas en l'analyse de leurs éléments [8]. Si la morale prescrit de ne pas sombrer dans l'alcoolisme, si la technique prescrit la quantité d'alcool à ingérer pour obtenir un taux d'alcoolémie donné dans le sang en fonction de la corpulence, c'est la prudence qui enseigne par l'expérience ce qu'il convient à chacun de boire et en quelles circonstances, pour accueillir, par exemple, des hôtes avec jovialité et humanité.

Le jugement de prudence est donc global et singulier quand le jugement technique est abstrait et universel. Prenant en considération tout ce qui fait qu'une situation est unique, il ne la détermine pas en la subsumant sous une loi universelle et il ne permet pas de lui appliquer des règles générales d'action. Pourtant, sans passer par une règle abstraite, il relie cette situation à des cas comparables et conseille une attitude en s'appuyant sur les similitudes et les dissemblances des cas. On peut donc tirer de l'expérience des conseils de prudence sans exiger au préalable la formulation d'une règle (comme en technique) ou d'un principe (comme en morale). 
Cette brève évocation de la prudence appelle deux remarques. La première : c'est bien de prudence que nous manquons, plutôt que de morale ou de technique, pour gérer les applications des découvertes scientifiques. Les intentions coupables sont rares ; les imprudences foisonnent, comme le montrent les affaires relatives à la politique sanitaire qui ont ému naguère l'opinion publique en France et dans d'autres pays. Dans l'affaire dite du " sang contaminé ", les responsables incriminés ont indiqué eux-mêmes par une lacune de leur défense le registre de leur responsabilité, puisqu'ils arguaient essentiellement d'une part, d'un point de vue technique, de l'impossibilité de connaître, à l'époque, le risque de développement d'un sida à partir de l'inoculation du virus par transfusion, d'autre part, d'un point de vue moral, de l'absence de toute intention de nuire. Pourtant, quel que soit le niveau des connaissances scientifiques et du pouvoir de la technique, la décision exige toujours de surcrôt, outre les bonnes intentions, l'aptitude proprement prudentielle à envisager une situation globalement, dans la totalité de ses développements possibles, et à trouver dans l'histoire des exemples comparables sur lesquels on peut s'appuyer pour déterminer au mieux une action salutaire. Le caractère exemplaire de cette affaire en a d'ailleurs fait depuis une référence en matière de politique sanitaire : la prudence se nourrit de l'expérience des imprudences passées.

La seconde remarque est la suivante : le jugement clinique est le jugement prudentiel par excellence. Certes, la médecine s'est dotée, durant les dernières décennies, d'une panoplie d'instruments techniques qui occupent un rôle important dans l'investigation des pathologies. Cependant, le jugement diagnostique et la décision thérapeutique ne se réduisent pas à un calcul à partir de ces données [9]. Il s'agit toujours pour le praticien de rassembler l'ensemble de ces éléments en les confrontant à l'expérience que constitue la consultation et le suivi médical de son patient. Au-delà, il n'est pas de bon diagnostic ni de bonne thérapeutique qui ne prenne en compte le mode de vie du malade, sa personnalité, son milieu. Il ne s'agit pas là seulement de facteurs subalternes dont l'effet se limiterait à une correction du jugement technique. Pour comprendre la raison du caractère nécessairement global et singulier du jugement médical, il faut porter son attention sur l'objet même de la médecine. En effet, la médecine n'est pas seulement l'art de guérir des maladies; elle est d'abord l'art de procurer la santé. Ces deux formules sont loin d'être équivalentes. La maladie est déterminable abstraitement. Elle se manifeste par une modification la plupart du temps quantifiable de certaines variables physiologiques. En agissant sur ses causes immédiates, on peut espérer supprimer le mal, et constater la guérison par un recouvrement des valeurs normales de grandeurs mesurables. Cependant, un tel schéma fait abstraction du caractère global et singulier de la vie d'un organisme humain. Comme l'a montré G. Canguilhem, " aucune guérison n'est retour à l'innocence biologique » [10]. La maladie imprime durablement sa marque dans l'organisme comme dans la personnalité du malade. On ne recouvre pas la santé d'avant l'affection, mais c'est une santé nouvelle qu'il faut construire, même lorsque la maladie ne laisse aucun handicap mesurable. $\mathrm{Si}$, à un certain niveau d'abstraction, les espèces de maladies sont semblables d'un patient à l'autre en sorte qu'elles peuvent être rangées dans une nosologie, il n'est de santé qu'individuelle ; c'est pourquoi la santé n'admet pas de définition abstraite. La santé dépend dès sa définition de l'usage qu'on en fait. Le technicien médical peut bien tracer des moyennes et des écarts types, il ne désigne pas ainsi une norme, s'il est vrai que la santé est l'aptitude du corps à transgresser les normes et à inventer de nouvelles normes relatives au mode de vie et au milieu de chaque personne. Cela vaut dans tous les registres : la santé n'a pas le même sens chez un athlète et chez un intellectuel parce que l'un et l'autre n'en attendent pas la même chose. Cela vaut peut-être particulièrement dans le registre de la sexualité. La santé, en la matière, ne peut être évaluée autrement que par la capacité à vivre, c'est-à-dire à inventer une vie sexuelle dans laquelle les émotions du corps épousent les émotions de l'âme jusqu'à la confusion. Ainsi, il y a des pathologies sexuelles mais il n'y a pas de sexualité normale. La médecine est donc un art qui connaît l'anormal mais ignore la norme. 
Rien ne me semble plus faux que cette remarque d'Aristote selon laquelle un médecin ne saurait soigner sans savoir d'abord ce qu'est la santé. La tâche de la médecine, des points de vue tant pratique que théorique, consiste à circonscrire de façon de plus en plus étroite les maladies et à élargir, dans le même temps, le champ de la santé jusqu'à la plus large amplitude.

C'est l'indétermination de la santé, sa perpétuelle singularisation, qui rend impossible la réduction du jugement médical à un calcul et rend irremplaçable la pratique clinique. Une médecine conçue comme art de la santé n'implique cependant pas que le médecin doive simplement accéder à la demande de son client car la demande n'est qu'un symptôme du malêtre et, à ce titre, elle exige une interprétation dans le cadre du jugement clinique, c'est-à-dire d'une confrontation à l'ensemble des autres symptômes. La suppression de la demande ne passe donc pas toujours par sa satisfaction directe : elle peut résulter de l'établissement d'un mode de vie plus satisfaisant, d'un nouvel état de santé que le médecin peut proposer dans un dialogue avec son patient. Les praticiens confrontés à la demande de somnifères savent ainsi parfois déjouer cette demande pour élaborer une hygiène de vie mieux adaptée aux rythmes physiologiques. La demande d'une amélioration des performances sexuelles ne peut être satisfaite médicalement qu'à la condition que cette satisfaction entraîne une meilleure santé, c'est-à-dire non pas la conformité à une norme universelle, non pas un équilibre statique, non pas un état de bonheur, mais une aptitude à inventer sa vie et à la vivre corps et âme confondus. La médicalité de l'acte peut donc ici servir de critère régulateur de l'intervention technique [11].

La médecine dispose ainsi de l'outil qui permet de juger de l'opportunité d'un traitement dans la singularité de chaque cas. La prise en compte de la personnalité du patient et de son mode de vie ne saurait être disjointe des autres aspects sémiotiques et des paramètres quantifiés que le médecin intègre dans le jugement clinique qui est un jugement de prudence conduisant à la décision thérapeutique. Mais, objectera-t-on, si le médecin est apte à juger de l'opportunité d'un traitement, il ne lui revient pas de fixer les valeurs qu'il convient de respecter dans ce jugement. Ne doit-il pas se tourner vers le moraliste et vers le législateur pour que l'un et l'autre lui assignent les cadres moraux et légaux de sa profession? Plus prosaïquement, l'absence de règle explicite ne risque-t-elle pas de laisser le médecin sans défense dans l'hypothèse d'une accusation devant les tribunaux?

L'histoire est ancienne. Un texte médical antique, datant d'une centaine d'années après Hippocrate, intitulé $L a$ loi [12], regrette déjà l'absence de réglementation de la profession médicale et en appelle aux juges pour départager les vrais médecins des charlatans qui s'affublent du titre de médecin sans l'être en fait. Or ce texte, postérieur au fameux Serment d'Hippocrate [13] (lequel est déjà plus tardif que l'époque d'Hippocrate), marque à mon sens le déclin de la médecine hippocratique. Précisément, les médecins formalisent les règles de la déontologie dans le Serment et en appellent au législateur dans $L a$ loi quand ils perdent le sens de leur entreprise [14]. Les textes anciens de la Collection hippocratique n'édictent pas de règle déontologique générale et ne font pas appel à des principes moraux universels. Ils témoignent d'un débat vivant, au sein de la profession médicale et face aux patients et à leur entourage, sur l'opportunité de telle ou telle pratique dans tel ou tel cas. Ce débat mêle des facteurs proprement cliniques (au sens moderne du terme) à des éléments relatifs à la personnalité du patient ou à la réputation de la profession dans l'opinion. Cette dimension éristique de la pratique médicale est tellement importante qu'un texte hippocratique définit le médecin par son aptitude à discuter avec à propos le traitement d'un confrère et à défendre opportunément ses propres prescriptions face aux accusations [15]. Jusqu'au Serment (exclu), la régulation de la pratique des médecins a trouvé dans le débat sur des cas singuliers une modalité proprement médicale. La déontologie n'était pas, au temps d'Hippocrate, une spécialité disjointe de la clinique.

Mais selon quelle valeur? La médecine n'estelle pas du moins contrainte d'importer des valeurs d'une société, d'une morale, d'une religion, d'une politique? Certes non, si l'on consi- 
dère l'idée avancée plus haut que la médecine n'est pas seulement l'art de soigner les maladies, mais d'abord celui de prodiguer la santé. La santé est donc la valeur à laquelle le médecin se voue. "La santé est le premier des biens ", proclame le traité hippocratique Des affections. Si la santé ne passe pas communément pour une valeur absolue ou pour une fin dernière, mais plutôt pour une condition nécessaire en vue de poursuivre des valeurs supérieures, c'est que, seule parmi les valeurs, elle ne produit pas de sens. Si cela donne un sens (peut-être illusoire?) à ma vie de poursuivre la justice, le bien ou la sainteté, cela n'offre pas de sens de poursuivre la santé, car la santé n'est que l'excellence de la vie elle-même. Or, cette vocation du médecin à promouvoir une valeur dénuée de sens est, du point de vue du moraliste, un scandale. Dès l'Antiquité, la figure d'Asclépios est celle d'un criminel accusé par Zeus d'avoir ressuscité, moyennant salaire, un homme dont le destin était achevé [16]. Depuis Platon jusqu'à Max Weber, des philosophes se sont émus de voir les médecins soigner des moribonds dont la vie n'aurait plus de sens [17]. Il est vrai que le médecin n'exige pas de son patient que celui-ci justifie sa vie et il ne lui propose pas une vie sensée. Il ne cherche que l'épanouissement de cette vie dans son envergure la plus large. Non seulement il laisse son patient libre de l'usage qu'il entend faire de sa vie, voire du sens qu'il lui confêre, mais toute l'activité du médecin consiste à restaurer et à amplifier cette aptitude à vivre en quoi réside proprement la santé.

Cette abstention du médecin à l'égard du sens ne doit pas être confondue avec une neutralité dans le domaine des valeurs. Le projet médical, depuis son origine hippocratique, est fondé sur la décision thérapeutique, c'est-à-dire, non pas la décision d'adopter tel ou tel traitement, mais la décision plus originelle de soigner les maux dont souffrent les hommes. Cette résolution définit l'attitude médicale comme une attitude éthique qui est différente de celle du prêtre ou du philosophe précisément en ce qu'elle s'attaque au mal sans recourir à l'artifice du sens. L'éthique médicale n'est donc pas une discipline auxiliaire de la médecine si l'on se réfère au projet fondateur de l'art médical ; à l'inverse, c'est l'ensemble des techniques dont le médecin se munit qui apparaît alors comme l'auxiliaire d'un projet originellement éthique.

Lorsque la profession médicale fait appel au moraliste et au législateur pour exiger des normes, des devoirs et des limites, elle s'écarte du projet médical comme projet éthique. Le médecin se place alors dans la position d'un technicien innocent, mettant à disposition son savoir et son savoir-faire, capable de modifier les fonctions vitales pour le pire ou le meilleur, selon des directives qui ne concerneraient plus la médecine elle-même. Le jugement clinique est alors réduit à sa dimension technique, et le client peut exiger du médecin qu'il accède à ses demandes comme un «distributeur de médicaments ", pourvu que sa demande n'excède pas les limites légales et morales toujours trop générales pour orienter la décision singulière du praticien.

À l'inverse, la relation du patient et du médecin doit être conçue comme un contrat en vue de la santé, lequel engage les deux parties à améliorer l'aptitude du patient à vivre plus amplement et plus longtemps. C'est dans le cadre de cet accord initial que le jugement clinique doit intégrer comme jugement prudentiel l'ensemble des aspects de la personne et des effets à long terme d'une action thérapeutique sur sa vie biologique et sa vie de relation. La mise en œuvre des techniques médicales dans le domaine de la sexualité peut donc trouver, dans cette perspective, une régulation éthique médicale.

Platon, disais-je en commençant, prête à Aristophane le mythe des couples fondus par l'amour en des boules indissociables. Il est aisé de montrer que l'état ainsi atteint par cette humanité mythique, en dépit de sa perfection, n'est pas la santé, car il lui manque l'amplitude de la vie, la faculté de s'adapter aux vicissitudes du milieu et celle de le transformer dans une vie de relation. Précisément parce que cet état est parfait, il est déterminé et définitif, tandis que la santé est un mouvement. Si cet état n'est pas la santé, le poursuivre ne relève pas de la médecine. L'interrogation sur la médicalité de l'acte permet donc d'orienter du point du vue éthique le jugement clinique et la thérapeutique. 


\section{Laurent Ayache}

Agrégé de philosophie, docteur, membre associé de l'unité de recherches "médecine grecque" (CNRS) et du Centre de recherches sur la philosophie antique (CNRS), professeur en classes préparatoires aux Écoles normales supérieures.

\section{REFERENCES}

1 PLATON, Banquet, $189 \mathrm{~d}-193 \mathrm{~d}$.

2 RAWLS J. , "Lidée d'un consensus par recoupement ", Revue de métaphysique et de morale, $1988, \mathrm{n}^{\circ}$ $1,3-32$, entend par là un accord sur des conceptions et des prescriptions pratiques dérivées de principes différents. Par exemple, on peut dériver une même définition de la mort ou un même statut de l'embryon à partir de conceptions divergentes de la vie humaine, et à partir de principes moraux ou religieux incompatibles entre eux.

3 GRMEK M., Histoire du sida. Début et origine d'une pandémie actuelle, Paris, 1989.

4 On peut remarquer que l'impératif moral proposé par H. Jonas, Le principe Responsabilité. Une éthique pour la civilisation technologique, 1979, trad. française par J. Greisch, Paris, 1990, exigeant la survie à long terme de l'humanité dans sa forme actuelle, n'est pas ici plus pertinent que les morales fondées sur le respect de la personne au présent.

5 BERNARD CL., Principes de médecine expérimentale, Paris, 1947, 1987, notamment pp. 32-35.

6 ARISTOTE, Éthique à Nicomaque, II, 6 et VI, 5-13. Voir P. Aubenque, La prudence chez Aristote, Paris, 1963, 1986 [troisième édition revue et augmentée d'un appendice sur La prudence chez Kant], P. Rodrigo, Aristote, l'eidétique et la phénoménologie, étude VII : «Prudence, convenance et situation ", pp. 123-136.

7 KANT E., Fondements de la métaphysique des mœurs, deuxième section.

8 KANT E., Anthropologie du point de vue pragmatique, Préface.

9 «I semble que malgré ses orientations de plus en plus techniques et le caractère de plus en plus scientifique de base de son exercice, la médecine ne puisse être assimilée à une science exacte et que la dimension de l'acte médical avec ses composantes individuelles, subjectives et psychologiques, ne puisse être réduite à l'application de formules ou de principes constants ", Dictionnaire de médecine Flammarion, Paris, 1975, 1989, s. v. "médecine".

10 CANGUILHEM G., Le normal et le pathologique, Paris, 1966, 1972.
11 Cette position differre de la "bioéthique " comprise comme une éthique dérivée des sciences de la vie en ce qu'elle fait, comme on le verra plus bas, de la décision thérapeutique (c'est-à-dire de la résolution de soigner les maux dont souffrent les hommes), et non de la normativité de la vie (laquelle demeure toujours indéterminée), le principe régulateur de l'acte médical. Sur les différentes acceptions de la bioéthique et sur l'éthique médicale en général, voir D. Folscheid, B. Feuillet-Le Mintier, J.-F. Mattei et J.-F. Mattéi [ces deux derniers auteurs étant presque homonymes], Philosophie, éthique et droit de la médecine, Paris, 1997.

12 Ce texte fait partie de la Collection hippocratique. On peut le lire dans l'édition de É. Littré, Euvres complètes d'Hippocrate en 10 volumes, Paris,1839-1861, tome IV, pp. 638-643. La Collection hippocratique est en cours d'édition et de traduction en français dans la Collection des Universités de France, aux Belles Lettres. Pour une introduction à son étude, voir J. Jouanna, Hippocrate, Paris, 1992 et, de façon plus modeste, L. Ayache, Hippocrate, "Que sais-je?", Paris, 1992.

13 Ibid., pp. 628-633. Sur le Serment, voir notamment Hippocrates, The Oath or The Hippocratic Oath, Introduction, greek text with facing english translation, commentary and interpretation by L. Edelstein with an appendix on The Hippocratic Patient and his Physician by H. N. Couch, Chicago, 1943.

14 AYACHE L., Médecine et sagesse. Essai sur la philosophie hippocratique, Paris, à paraître.

15 MALADIES I, É. LITTRÉ, Op. cit., tome VI, pp. 138205. L'édition la plus récente est : Hippocrates, with an english translation by P. Potter, The Lœb Classical Library, Cambridge, Massachussets et London, vol. V, 1988.

16 PINDARE, IIIe Pythique, 3. Cf. Platon, République, III, 408 b-c.

17 PLATON, République, III, 408 b-c ; M. Weber, Le savant et le politique, Paris, 1959, p. 78. 